\title{
Nuevos registros de opistobranquios en el Parque Nacional Sistema Arrecifal Veracruzano, México
}

\section{New opisthobranch records in the Veracruz Reef System National Park, Mexico}

\author{
Andrea Zamora-Silva ${ }^{\circledR}$ y Deneb Ortigosa ${ }^{2}$ \\ ${ }^{1}$ Phylogenetic Systematics and Evolution Research Group, University Museum of Bergen, Natural History Collections, University of Bergen, PB 7800, \\ 5020-Bergen, Norway. \\ ${ }^{2}$ Facultad de Ciencias, Universidad Nacional Autónoma de México. Apartado postal 70-399, 04510, México, D. F., México. \\ \Andrea.Zamora@bm.uib.no
}

\begin{abstract}
Resumen. Diversos trabajos se han realizado sobre la riqueza y abundancia de moluscos en los arrecifes del litoral costero del golfo de México, pero éstos se han enfocado principalmente en el conocimiento de las especies de gasterópodos prosobranquios y bivalvos y sólo se han registrado 6 especies de opistobranquios de este ecosistema. En este trabajo se presenta una lista de 23 especies de opistobranquios del Parque Nacional Sistema Arrecifal Veracruzano, que es un complejo de 23 formaciones coralinas ubicadas en la costa del estado de Veracruz en el golfo de México. La lista incluye 9 registros nuevos e imágenes de varias de las especies aquí citadas.
\end{abstract}

Palabras clave: Veracruz, opistobranquios, sistemática, nuevos registros.

\begin{abstract}
Several studies have been conducted on the richness and abundance of molluscs in the coastal coral reefs of the Gulf of Mexico since 1960's, but these have focused mainly on knowledge of prosobranch gastropods and bivalves species, while only 6 species of opisthobranchs have been registered for this ecosystem. In this paper we present a list of 23 species of opisthobranchs from the Veracruz Reef System National Park, a complex of 23 coral formations located on the coast of Veracruz on the Gulf of Mexico. The list includes 9 new records, a systematic arrangement and pictures of several of the species mentioned here.
\end{abstract}

Key words: Veracruz, opisthobranchs, systematics, new records.

\section{Introducción}

El Parque Nacional Sistema Arrecifal Veracruzano (PNSAV) es un complejo formado por 23 arrecifes coralinos situados en la costa veracruzana del golfo de México. El PNSAV posee 2 agrupaciones de arrecifes: una al norte frente al puerto de Veracruz y otra al sur frente a la población de Antón Lizardo (Fig. 1). En 1992 fue decretado Parque Nacional (Secretaría de Desarrollo Social, 1992); sin embargo, el constante hundimiento de bloques de concreto para extender la superficie portuaria, la extracción excesiva de especies para uso artesanal o académico, el uso del coral como material de construcción, el vertimiento de residuos sólidos y el impacto turístico han diezmado las comunidades de este importante ecosistema. Diversos autores han sugerido monitoreos periódicos de los impactos antropogénicos, revisión de los programas de extracción de especies y evaluación de la continuidad, recuperación o pérdida de las especies residentes dentro

Recibido: 15 octubre 2010; 25 noviembre 2011 del Parque (Vargas-Hernández et al., 1993). Aunque la investigación malacológica en el PNSAV se desarrolla desde la década de los 60 , sólo se conocen 6 especies de opistobranquios para este sistema arrecifal; 3 pueden ser vistas fácilmente en casi todas las estaciones del año (Elysia crispata, Aplysia dactylomela y A. brasiliana) y el resto pueden identificarse por medio de su concha (Japonacteon punctostriatus, Acteocina candei y Bulla occidentalis; obs. pers.). Por el bajo número de especies registradas, el inventario de opistobranquios del PNSAV se considera incompleto, lo cual exhibe la necesidad de estudios que indiquen el estado actual de la biodiversidad de este grupo de moluscos. El monitoreo de las especies de opistobranquios en el PNSAV fue el principal objetivo de este trabajo.

A los opistobranquios se les conoce comúnmente con el nombre de babosas marinas. Estos moluscos gasterópodos presentan coloraciones vistosas, cuerpos y estructuras externas variadas. En la actualidad se han descrito aproximadamente 6000 especies en el mundo (Camacho-García, 2009). En México se han registrado alrededor de 400, principalmente en las costas del océano Pacífico (Angulo- 


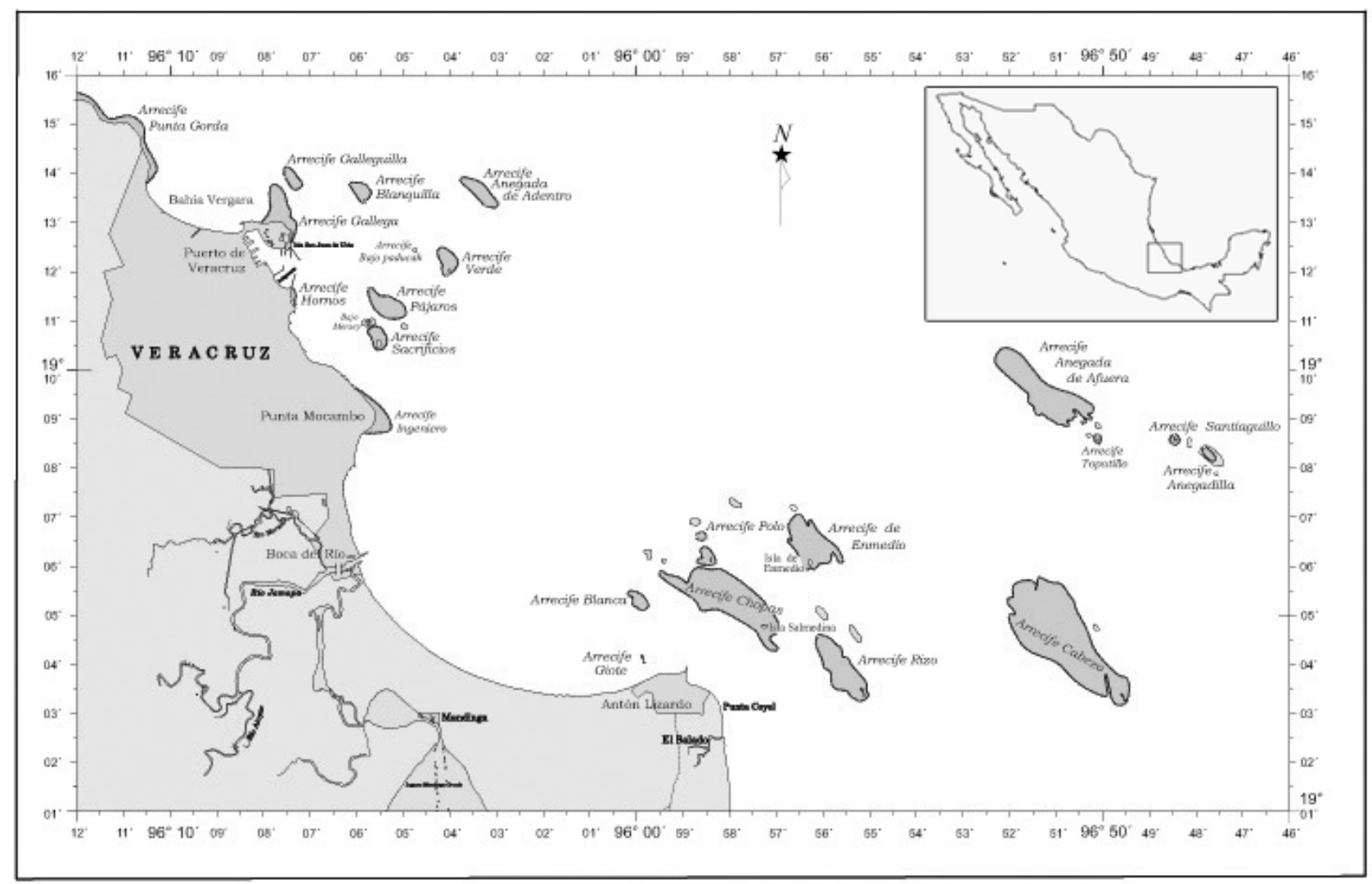

Figura 1. Arrecifes del Parque Nacional Sistema Arrecifal Veracruzano (PNSAV) mostrando el grupo norte (Gallega, Galleguilla, Anegada de Adentro, La Blanquilla, isla Verde, isla Sacrificios, Pájaros, Hornos, Ingeniero, Punta Mocambo y Punta Gorda) y el grupo sur (Giote, Polo, Blanca, Punta Coyol, Chopas, Enmedio, Polo, Cabezo, El Rizo, Santiaguillo, Anegada de Afuera, Anegadilla y Topatillo).

Campillo, 2002; Hermosillo et al., 2006; Camacho-García, 2009). A diferencia del resto de los gasterópodos, los opistobranquios exhiben una tendencia evolutiva hacia la pérdida, reducción o internalización de la concha, aunque la mayoría de las especies carecen de ella o la pierden al llegar a su etapa adulta. Este proceso ha originado una gran diversidad de formas, colores, adaptaciones a ambientes, técnicas de caza, de alimentación y de mimetismo (Thompson, 1976).

El estudio de la biodiversidad de los opistobranquios del PNSAV es relevante si consideramos que estos organismos: $a$ ), son componentes importantes de una comunidad arrecifal; $b$ ), participan en las redes tróficas siendo depredadores activos de invertebrados y peces pequeños; c), algunas especies establecen relaciones simbióticas con algas y protozoos para generar fuentes metabólicas alternas y $d$ ), pueden ser fuente natural de sustancias útiles en farmacolgía (e.g. los metabolitos secundarios de algunas especies de los géneros Aglaja, Navanax y Philinopsis, con efectos citotóxicos y antibióticos [Ávila, 1992]).
Las áreas marinas protegidas, como el PNSAV, son herramientas efectivas para conservar un arrecife de coral y sus comunidades residentes. La riqueza y abundancia de especies de invertebrados marinos representan un importante factor biótico que sin duda debe ser considerado en las estrategias de manejo y conservación de un arrecife coralino (NOAA, 2011).

\section{Materiales y métodos}

Desde marzo de 2002 hasta mayo de 2005 se realizaron muestreos ocasionales en los arrecifes Ingeniero, Gallega, Galleguilla y en las islas Verde y Sacrificios, todos ellos parte del PNSAV y ubicados en el grupo norte del Parque (Permiso de colecta DGOPA0790). La recolecta de ejemplares se realizó en cada uno de los sustratos de la laguna y del talud arrecifal (arena, pastos marinos, coral y algas). Los organismos fueron narcotizados con cloruro de magnesio al $2 \%$ disuelto en agua dulce, fijados en formol al $4 \%$ y posteriormente conservados en alcohol al $70 \%$. Todo el material biológico recolectado se depositó en la 
Colección Nacional de Moluscos (Instituto de Biología) y en la Colección Malacológica "Dr. Antonio García-Cubas" (Instituto de Ciencias del Mar y Limnología, Campus Ciudad Universitaria), ambas colecciones pertenecientes a la Universidad Nacional Autónoma de México (UNAM). Finalmente, se realizó una revisión bibliográfica para localizar registros previos.

\section{Resultados}

Se recolectaron 647 organismos que corresponden a 16 especies. La revisión bibliográfica permitió incluir 7 especies más para el PNSAV (Cuadro 1). Los sustratos en los que se encontraron las especies fueron: pastos marinos de Thalassia testudinum, algas verdes (Padina variegata, Caulerpa sertularoides y Ulva fasciata), algas pardas (Gracillaria blodgetti), sargazo marino, coral, arena y sustrato rocoso. En el Cuadro 2 puede verse un resumen de las especies localizadas por sustrato, así como su estacionalidad y hábitos alimenticios. A continuación se muestra la lista sistemática con sinónimos de las especies, según las categorías propuestas por Bouchet y Rocroi (2005):

Clado Heterobranchia

Grupo informal "Lower Heterobranchia"

Cuadro 1. Lista de especies de opistobranquios registradas en algunos arrecifes del Parque Nacional Sistema Arrecifal Veracruzano

\begin{tabular}{|c|c|c|c|c|c|c|c|}
\hline Especies & 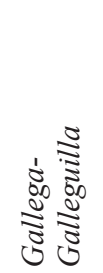 & $\begin{array}{l}\frac{\pi}{2} \\
\frac{5}{2} \\
\frac{5}{2}\end{array}$ & 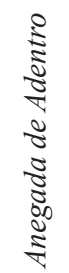 & 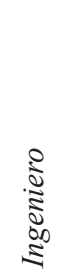 & 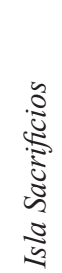 & 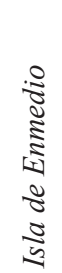 & 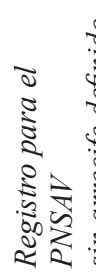 \\
\hline
\end{tabular}

Japonacteon punctostriatus (C. B. Adams, 1840)

Ringicula semistriata d'Orbigny, 1842

Bulla occidentalis Adams, 1850

Haminoea antillarum (d'Orbigny 1841)

Haminoea elegans (Gray, 1825)

Haminoea succinea (Conrad, 1846)

Navanax aenigmaticus (Bergh, 1893)

Acteocina canaliculata (Say, 1826)

Acteocina candei (d'Orbigny, 1842)

Volvulella persimilis (Mörch, 1875)

Aplysia brasiliana Rang, 1828

Aplysia dactylomela Rang, 1828

Aplysia juliana Quoy y Gaimard, 1832

Bursatella leachii pleii Rang, 1828

Stylocheilus longicaudus (Quoy y Gaimard, 1894)

Stylocheilus striatus (Quoy y Gaimard, 1832)

Dolabrifera dolabrifera (Cuvier, 1817)

Oxynoe antillarum Mörch, 1863

Elysia subornata Verrill, 1901

Elysia crispata Mörch, 1863

Discodoris evelinae Er. Marcus, 1955

Spurilla neapolitana (delle Chiaje, 1841)

Dondice occidentalis (Engel, 1925)

* Registros nuevos con negritas. 
Superfamilia Acteonoidea d'Orbigny, 1843

Familia Acteonidae d'Orbigny, 1843

Japonacteon punctostriatus (C. B. Adams, 1840): Acteon punctostriatus (C. B. Adams, 1840); Tornatella punctostriata C. B. Adams, 1840; Rictaxis punctostriatus (C. B. Adams, 1840); Actaeon punctatus (d'Orbigny, 1841); Tornatella punctata d'Orbigny, 1841; Actaeon floridanus Conrad, 1846; Actaeon cubensis Gabb, 1873; Actaeon riomaensis Maury, 1917.

Distribución. EUA: Massachusetts, New York, New Jersey, Maryland, Virginia, North Carolina South Carolina, Florida, Texas; México: Veracruz, Yucatán; Costa Rica, Panamá, Colombia, Venezuela, Bermudas, Cuba, Jamaica, República Dominicana, Puerto Rico, islas Vírgenes, Guadeloupe y Martinica (Rice y Kornicker, 1962; Vokes y Vokes, 1983; García-Cubas y Reguero, 1990; Reguero y García-Cubas, 1993; Pérez-Rodríguez, 1997; Valdés et al., 2006; Rosenberg, 2009).

Superfamilia Ringiculoidea Philippi, 1853

Familia Ringiculidae Philippi, 1853

Ringicula semistriata d'Orbigny, 1842: Ringicula semistriata d'Orbigny, 1842; Ringicula tridentata Guppy, 1873; Ringicula hypograpta Brown y Pilsbry, 1913.

Distribución. EUA: North Carolina, Florida, Louisiana, Texas; México: Campeche, Yucatán; Colombia, Jamaica, Puerto Rico (Rice y Kornicker, 1965; Rosenberg, 2009).

Grupo informal Opisthobranchia Milne-Edwards, 1848*

Clado Cephalaspidea Fischer, P., 1883

Superfamilia Bulloidea Gray, 1827

Familia Bullidae Gray, 1827

Bulla occidentalis Adams, 1850 (Fig. 2E): Bulla striata Bruguière, 1792; Bulla umbilicata Röding, 1798; Bulla media Philippi, 1847; Bulla rubiginosa Gould, 1852; Bulla (Bullea) nux Menke, 1853; Bulla (Bullea) sulcata Menke, 1853; Bulla amygdala Pilsbry, 1895; Bulla occidentalis Peile, 1926.

Distribución. EUA: Florida, Texas; México, Belice, Costa Rica, Panamá, Bahamas, Antillas, Cuba, Jamaica, Puerto Rico, Trinidad, Surinam, Colombia, Venezuela, Brasil y Uruguay (Malaquias y Reid, 2008).

Superfamilia Haminoeoidea Pilsbry, 1895

Familia Haminoeidae Pilsbry, 1895

Subfamilia Haminoeinae Pilsbry, 1895

Haminoea antillarum (d'Orbigny, 1841) (Fig. 2B): Bulla antillarum d'Orbigny, 1841; Bulla cerina Menke, 1853; Haminea guadaloupensis Sowerby II, 1868.

Distribución. EUA: Florida, Texas; México: Veracruz, Campeche, Quintana Roo; Costa Rica, Panamá, Colombia, Curaçao, Bonaire, Venezuela, Bermudas; Cuba: La
Habana; Jamaica, Puerto Rico, Santa Cruz, Granada, Guadeloupe; Brasil: Pernambuco, Rio de Janeiro, São Paulo, Rio Grande do Sul (Andrews, 1971; Ekdale, 1974; García-Cubas et al., 1990; de la Cruz-Francisco y González-Gándara, 2007; Valdés et al., 2006; Rosenberg, 2009).

Haminoea elegans (Gray, 1825): Bulla elegans Gray, 1825; Bullaea guildingii Swainson, 1840; Bulla diaphana Gould, 1852; Haminoea taylorae Petuch, 1987; Haminoea guadaloupensis Sowerby II, 1868.

Distribución. EUA: Florida, Texas; México: Veracruz, Tabasco, Quintana Roo; Panamá, Trinidad y Tobago, Costa Rica, Colombia, Curaçao, Bonaire, Venezuela, Bermudas, Cuba, islas Caimán, St. Croix, St. Vicente, islas Granada, Jamaica, Puerto Rico, Martinica, Santa Lucía, Granada, Santo Tomás, Santa Cruz; Brasil: Alagoas (Vokes y Vokes, 1983; de la Cruz-Francisco y González-Gándara, 2007 y Valdés et al., 2006; Rosenberg, 2009).

Haminoea succinea (Conrad, 1846): Bulla succinea Conrad, 1846; Haminoea solidior Vanatta, 1901.

Distribución. EUA: Florida, Louisiana, Texas; México: Veracruz, Campeche, Yucatán, Quintana Roo; Colombia, Venezuela: Sucre, isla Margarita; Bermudas, Puerto Rico, San Martín, San Bartolomé (Rice y Kornicker; 1962; Andrews, 1971; Vokes y Vokes, 1983, Cruz-Ábrego et al., 1994; De la Cruz-Francisco y González-Gándara, 2007; Valdés et al., 2006; Rosenberg, 2009).

Superfamilia Philinoidea Gray, 1850

Familia Aglajidae Pilsbry, 1895

Navanax aenigmaticus (Bergh, 1893) (Fig. 2C): Posterobranchaea maculata d'Orbigny, 1835; Doridium gemmatum Mörch, 1863; Aglaja gemmatus (Mörch, 1863); Posterobranchus orbignyanus Rochebrune, 1882; Navarchus aenigmaticus Bergh, 1893; Doridium punctilucens Bergh, 1893; Aglaja punctilucens (Bergh, 1893); Chelidonura africana Pruvot-Fol, 1953; Chelidonura evelinae Er. Marcus, 1955; Aglaja evelinae (Er. Marcus, 1955); Chelidonura nyanyana Edmunds, 1968; Chelidonura dica Er. Marcus y Ev. Marcus, 1970; Aglaja dica (Er. Marcus y Ev. Marcus, 1970); Chelidonura sabina Er. Marcus y Ev. Marcus, 1970.

Distribución. EUA: Florida; Belice, Honduras, Costa Rica, Colombia, Venezuela, Bahamas, Curaçao, Jamaica, islas Vírgenes, Guadeloupe, Martinica, Barbados, San Bartolomé, San Vicente, Granada, Brasil (Valdés et al., 2006).

Familia Cylichnidae H. Adams y A. Adams, 1854.

Acteocina canaliculata (Say, 1826) (Fig. 2A): Volvaria canaliculata Say, 1826; Tornatina canaliculata (Say, 1826); Retusa canaliculata (Say, 1826); Bullina canaliculata (Say, 1826); Utriculus canaliculata (Say, 1826); Utriculastra canaliculata (Say, 1826); Acteon wetherwi- 
Cuadro 2. Opistobranquios presentes en el Parque Nacional Sistema Arrecifal Veracruzano, según el tipo de sustrato y alimentación *

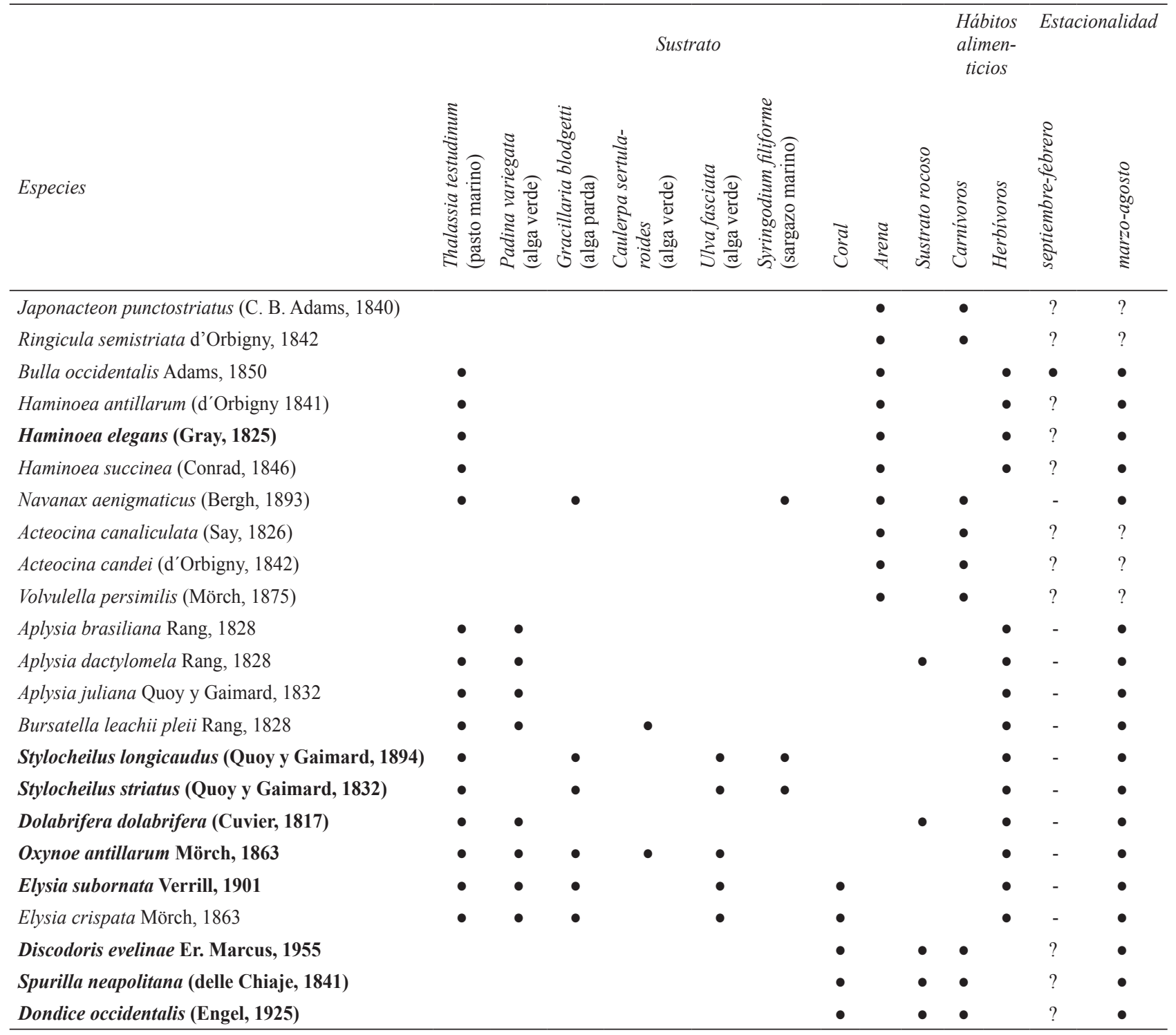

* Las especies con negritas son registros nuevos

lli I. Lea, 1833; Bulla obstricta Gould, 1839; Cylichnella candei auct. non d'Orbigny, 1841; Bulla incincta Mighels, 1844; Acteocina chowanensis Richards, 1947; Bullina wetherwilli (I. Lea, 1833); Bulla obstricta Gould, 1839.

Distribución. Canadá: Gulf of Sanint Lawrence, New Scotland, Prince Edward Island, New Brunswick; EUA: Maine, Massachussets, Rhode Island, Connecticut, New York, New Jersey, Maryland, Virginia, North Carolina, South Carolina, Florida, Georgia, Louisiana, Texas; México: Tamaulipas, Tabasco, Veracruz, Campeche, arrecife Alacranes (FloresAndolais et al., 1988; Reguero y García-Cubas, 1989; García-Cubas et al., 1990; García-Cubas y Reguero, 1990;
García-Cubas et al., 1992; Reguero y García-Cubas, 1993; Valdés et al., 2006; Rosenberg, 2009).

Acteocina candei (d'Orbigny, 1842): Tornatina canaliculata auct. non Say, 1822; Bulla pusilla Pfeiffer, 1840; Retusa candei (d'Orbigny, 1841); Bulla candei d'Orbigny, 1841; Tornatina candei (d'Orbigny, 1841); Cylichna virginica Conrad, 1868; Acteocina anetaspira Woodring, 1928; Acteocina cederstromi Richards, 1947; Acteocina vaughani Gardner, 1948; Acteocina elachista Woodring, 1970. Distribución. EUA: North Carolina, Florida, Texas; México: Campeche, Yucatán, arrecife Alacranes, Quintana Roo, Cozumel; Costa Rica, Panamá, Colombia; 

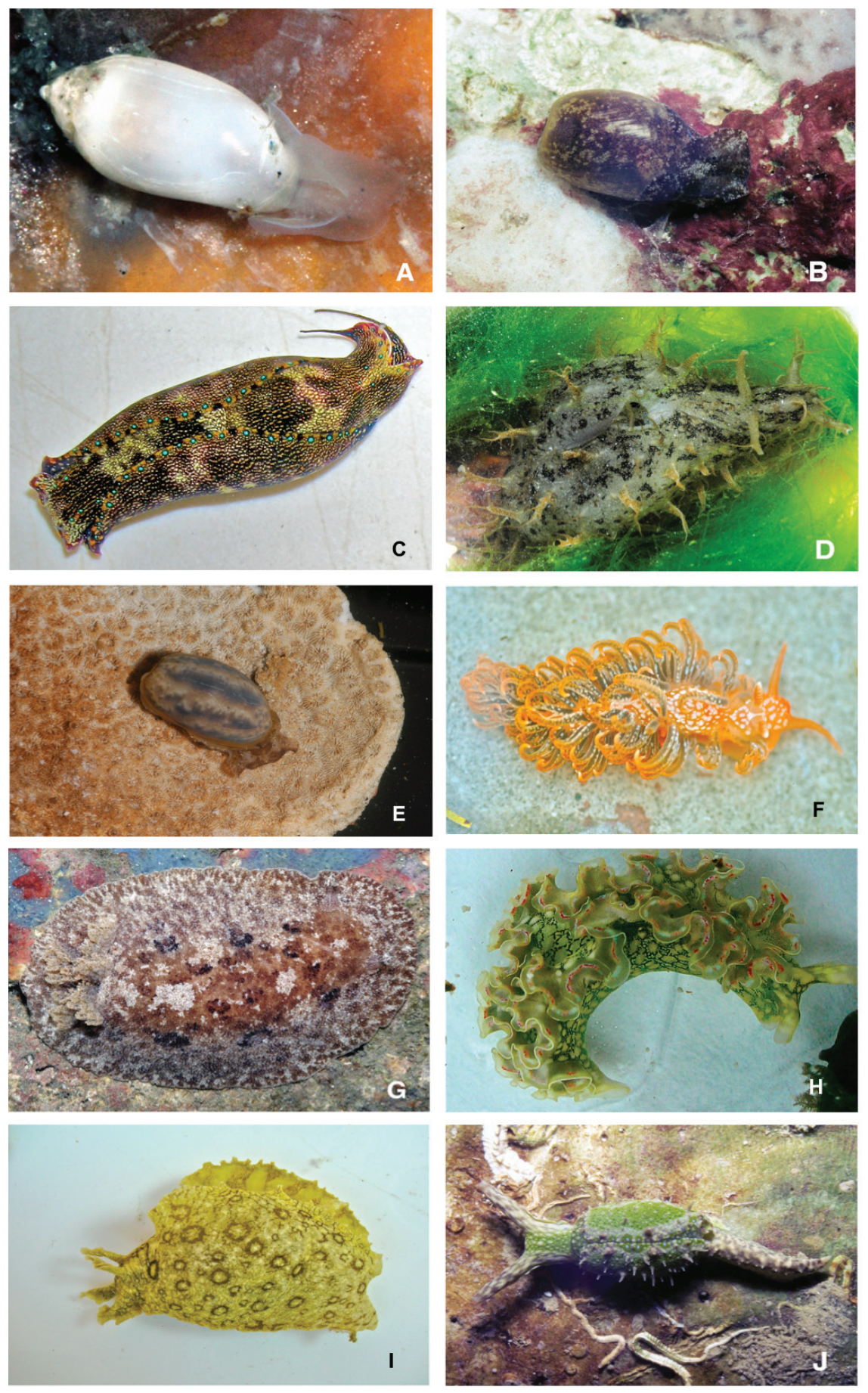

Figura 2. Algunas de las especies de opistobranquios del PNSAV. A, Acteocina canaliculata (Say, 1826); B, Haminoea antillarum (d'Orbigny 1841); C, Navanax aenigmaticus (Bergh, 1893); D, Bursatella leachii pleii de Blainville, 1817; E, Bulla occidentalis Adams, 1850; F, Spurilla neapolitana (delle Chiaje, 1841); G, Discodoris evelinae Er. Marcus, 1955; H, Elysia crispata Mörch, 1863; I, Aplysia dactylomela Rang, 1828 y J, Oxynoe antillarum Mörch, 1863. Fotos A, B, D, G y J: Ángel Valdés; E: Manuel Melquias; C, F, H e I: Andrea Zamora. 
Venezuela: Zulia, Falcón; Bermudas, Cuba, Jamaica, Puerto Rico; islas Vírgenes: Santo Tomás, Santa Cruz; Guadeloupe, Martinica, Guyana; Brasil: Pernambuco; Argentina: Buenos Aires (Cruz-Ábrego et al., 1994; Valdés et al., 2006; Rosenberg, 2009.)

Familia Retusidae Thiele, 1925.

Volvulella persimilis (Mörch, 1875): Volvula cylindrica Gabb, 1873; Volvula persimilis Mörch, 1875; Rhizorus persimilis (Mörch, 1875); Volvula oxytata Bush, 1885; Retusa oxytata (Bush, 1885); Rhizorus oxytata (Bush, 1885); Volvula cercadensis Van Winkle, 1921; Volvulella moerchi Dall, 1927; Volvula ischnatracta Pilsbry, 1930. Distribución. EUA: North Carolina, Florida, Louisiana, Texas; México: Yucatán, Quintana Roo; Costa Rica, Colombia, Venezuela: Falcón, Sucre, isla Margarita; Bermudas, Cuba, Surinam; Brasil: Pernambuco, Alagoas, São Paulo, Rio Grande do Sul; Uruguay (Andrews, 1971).

Clado Aplysiomorpha Rafinesque, 1815

Superfamilia Aplysioidea Lamarck, 1809

Familia Aplysiidae Lamarck, 1809

Subfamilia Aplysiinae Lamarck, 1809

Aplysia brasiliana Rang, 1828: Laplysia fasciata Poiret, 1789; Tethys brasiliana (Rang, 1828); Aplysia lurida d'Orbigny, 1835; Syphonota lurida (d'Orbigny, 1835); Tethys livida (d'Orbigny, 1837); Aplysia livida d'Orbigny, 1837; Aplysia cailleti Deshayes, 1857; Aplysia guadaloupensis Sowerby II, 1869; Tethys floridensis Pilsbry, 1895.

Distribución. EUA: New Jersey, Florida, Texas; México: Veracruz; Costa Rica, Colombia, Aruba, Venezuela: Sucre; Bermudas; Brasil: Santa Catarina (Strenth y Blankenship, 1977; Wiley et al., 1982; de la CruzFrancisco y González-Gándara, 2007; Zamora-Silva y Naranjo-García, 2008).

A. dactylomela Rang, 1828 (Fig. 2I): Aplysia argus Rüppell y Leuckart, 1828; Tethys dactylomela (Rang, 1828); Aplysia dactylomela Rang, 1828; Aplysia protea Rang, 1828 ; Aplysia tigrina Rang, 1828 ; Aplysia radiata Ehrenberg, 1831; Aplysia scutellata Ehrenberg, 1831; Aplysia ocellata d'Orbigny, 1839; Aplysia fimbriata Adams y Reeve, 1850; Aplysia schrammi Deshayes, 1857; Syphonota viridescens Pease, 1868; Aplysia angasi Sowerby II, 1869; Aplysia aequorea Heilprin, 1888; Tethys panamensis Pilsbry, 1895; Aplysia benedicti Eliot, 1899; Aplysia megaptera A. E. Verrill, 1900; Tethys megaptera (A. E. Verrill, 1900); Aplysia velifer Bergh, 1905; Aplysia operta Burne, 1906; Aplysia odorata Risbec, 1928; Aplysia annulifera Thiele, 1930; Aplysia bourailli Risbec, 1951.

Distribución. EUA: New Jersey, Florida, Texas; México: Veracruz, Quintana Roo; Belice, Honduras, Costa Rica,
Panamá, Colombia, Aruba, Curaçao, Bonaire; Venezuela: archipiélago de Los Roques; Bermudas; islas Caimán: Little Cayman; Jamaica, Puerto Rico, islas Vírgenes: San Juan, Anguilla; Guadeloupe: San Martín, San Christopher, Barbados, Brasil: Ceara, Rio Grande do Norte, Pernambuco, Rio de Janeiro y São Paulo (Moore, 1958; Andrews, 1971; Strenth y Blankenship, 1977; Quintana, 1991; Redfern, 2001; de la Cruz-Francisco y GonzálezGándara, 2007; Valdés et al., 2006; Zamora-Silva y Naranjo-García, 2008; Rosenberg, 2009).

A. juliana Quoy y Gaimard, 1832: Aplysia sorex auct. non Rang, 1828; Aplysia juliana Quoy y Gaimard, 1832; Aplysia rangiana d'Orbigny, 1835; Syphonota bipes Pease, 1860; Aplysia sandvichensis Sowerby II, 1869; Aplysia sibogae Bergh, 1905; Aplysia woodii Bergh, 1908; Tethys capensis O'Donoghue, 1929; Aplysia petiti Risbec, 1929; Aplysia badistes Pilsbry, 1951; Aplysia parva Pruvot-Fol, 1953; Aplysia quoyana Engels y Eales, 1957.

Distribución. EUA: Florida; Curaçao, Barbados; Brasil: Pernambuco, São Paulo (Valdés et al., 2006).

Bursatella leachii pleii Rang, 1828 (Fig. 2D): Bursatella leachii Blainville, 1817; Bursatella savignyiana Audouin, 1826; Aplysia pleii Rang, 1828; Aclesia pleii (Rang, 1828); Bursatella lacinulata Gould, 1852.

Distribución. EUA: North Carolina, Florida, Texas; México: Veracruz; Belice, Costa Rica, Panamá, Colombia, Venezuela, islas Vírgenes, Jamaica, Aruba, Curaçao, Bonaire (Rosenberg, 2009), Bermudas, Puerto Rico, Trinidad y Tobago; Brasil: Pernambuco, Rio de Janeiro, São Paulo, Rio Grande do Sul (Andrews, 1971; Strenth y Blankenship, 1977; Pérez-Rodríguez, 1997; de la CruzFrancisco y González-Gándara, 2007; Valdés et al., 2006; Rosenberg, 2009).

Stylocheilus longicaudus (Quoy y Gaimard, 1824): Aplysia longicauda auct. non Quoy y Gaimard, 1824; Aclesia longicauda auct. non Quoy y Gaimard, 1824; Notarchus citrinus Rang, 1828; Aplysia nudata Rang, 1828; Placobranchus ocellatus Rang, 1828; Aplysia striata Quoy y Gaimard, 1832; Aplysia euchlora A. Adams, 1861; Notarchus polyomma Mörch, 1863; Aclesia polyomma (Mörch, 1863); Tethys adamsi Pilsbry, 1896.

Distribución. Circuntropical. África, mar Rojo; Ecuador: Galápagos; EUA: Hawai, Florida; México: Baja California; Brasil (Valdés et al., 2006; Gosliner et al., 2008).

Stylocheilus striatus (Quoy y Gaimard, 1832): Aplysia longicauda auct. non Quoy y Gaimard, 1824; Aclesia longicauda auct. non Quoy y Gaimard, 1824; Aplysia striata Quoy y Gaimard, 1832; Notarchus polyomma Mörch, 1863; Aclesia polyomma (Mörch, 1863).

Distribución. EUA: Florida, Dry Tortugas; Belice, Colombia, Venezuela, Aruba, Curaçao, Bonaire, Bermudas, 
Bahamas, islas Caimán, Jamaica, Puerto Rico, islas Vírgenes, Martinica, Barbados, San Vicente y Las Granadinas, Granada; Brasil (Valdés et al., 2006).

Dolabrifera dolabrifera (Cuvier, 1817) (Fig. 2M): Aplysia ascifera Rang, 1828; Aplysia dolabrifera Rang, 1828; Dolabrifera cuvieri H. y A. Adams, 1854; Dolabrifera maillardi Deshayes, 1863; Dolabrifera sowerbyi Sowerby II, 1868; Dolabrifera nicaraguana Pilsbry, 1896; Dolabrifera swiftii Pilsbry, 1896; Dolabrifera virens A. E. Verrill, 1901.

Distribución. EUA: Florida; Costa Rica, Colombia; Venezuela: archipiélago de Los Roques; Aruba, Curaçao, Bonaire, Bermudas, Bikini, islas Caimán, Puerto Rico, Jamaica, islas Vírgenes, Saba, Antigua, Martinique, Santa Lucía, Barbados, Granada; Brasil: Rio de Janeiro (Valdés et al., 2006).

Clado Sacoglossa von Ihering, 1876

Subclado Oxynoacea

Superfamilia Oxynooidea Stoliczka, 1868

Familia Oxynoidae Stoliczka, 1868

Oxynoe antillarum Mörch, 1863 (Fig. 2J): Oxynoe aguayoi Jaume, 1945; Lophocercus antillarum (Mörch, 1863).

Distribución. EUA: Florida; México: Yucatán; Belice, Honduras, Costa Rica, Panamá, Venezuela, Bahamas, Curaçao, Bermudas, islas Caimán, Jamaica, Dominicana, Puerto Rico, islas Vírgenes, Martinica, Santa Lucía, Barbados, San Vicente y Las Granadinas, Granada, Trinidad y Tobago; Brasil (Vokes y Vokes, 1983; sinónimos según Valdés et al., 2006).

Subclado Placobranchacea

Superfamilia Placobranchoidea Gray, 1840

Familia Placobranchidae Gray, 1840

Elysia subornata Verrill, 1901: Elysia subornata A. E. Verrill, 1901; Elysia cauze Er. Marcus, 1957.

Distribución. EUA: Florida; México: Veracruz, Quintana Roo; Belice, Bermuda, Bahamas, Aruba, islas Caimán, Jamaica, Puerto Rico, islas Vírgenes, Martinica, Granada, Trinidad y Tobago; Brasil: São Paulo. (de la Cruz-Francisco y González-Gándara, 2007; Valdés et al., 2006; Rosenberg, 2009).

Elysia crispata Mörch, 1863 (Fig. 2H): Tridachia ornata auct. non Pease, 1860; Tridachia crispata (Mörch, 1863); Elysia schiadura Mörch, 1863; Elysia (Tridachia) crispata Mörch, 1863; Tridachia schrammi (Mörch, 1863); Elysia verrilli Pruvot-Fol, 1946; Elysia (Elysiopterus) pruvotfolae Er. Marcus, 1957; Tridachia whitae Er. Marcus, 1957; Elysia clarki Pierce et al., 2006.

Distribución. EUA: Florida, Dry Tortugas; México: Veracruz, Quintana Roo; Belice, Honduras, Costa Rica, Colombia, Venezuela, Bermuda, islas Caimán, Bahamas,
Aruba, Curaçao, Bonaire, Jamaica, Haití, Puerto Rico, islas Vírgenes, San Martín, Antigua, Santa Lucía, Martinica, Guadeloupe, Turku y Caicos, San Vicente y Las Granadinas, Barbados, Trinidad y Tobago (de la Cruz-Francisco y González-Gándara, 2007; Valdés et al., 2006).

Clado Nudibranchia

Subclado Gnathodoridacea

Superfamilia Doridoidea Rafinesque, 1891

Familia Discodoridae Bergh, 1891

Discodoris evelinae Er. Marcus, 1955 (Fig. 2G): Discodoris hedgpethi Ev. Marcus y Er. Marcus, 1960; Discodoris spetteda Ev. Marcus y Er. Marcus, 1966.

Distribución. EUA: Florida, Texas; Costa Rica, Honduras, Panamá, Colombia, Venezuela, Bahamas, islas Caimán, Puerto Rico, Jamaica, Barbados, Martinica, Santa Lucía, Guadeloupe, San Vicente y Las Granadinas; Brasil (Valdés et al., 2006).

Subclado Aeolidida

Superfamilia Aeolidioidea Gray, 1827

Familia Aeolidiidae Gray, 1827

Spurilla neapolitana (delle Chiaje, 1841) (Fig. 2F): Eolidia neapolitana delle Chiaje, 1841; Flabellina inornata A. Costa, 1866; Eolis conspersa P. Fischer, 1869; Spurilla braziliana MacFarland, 1909; Eolidina gabriellae Vannucci, 1952; Spurilla dakariensis Pruvot-Fol, 1953; Spurilla mograbina Pruvot-Fol, 1953.

Distribución. EUA: Florida, Texas; Belice, Honduras, Costa Rica, Colombia, Venezuela, Bahamas, Curaçao, Bermudas, islas Vírgenes, Jamaica, Puerto Rico, Barbados, San Vicente y Las Granadinas; Brasil (Valdés et al., 2006).

Familia Facelinidae Bergh, 1889

Dondice occidentalis (Engel, 1925): (Fig. 2I): Facelina bostoniensis auct. non Couthouy, 1838; Caloria occidentalis Engel, 1925; Facelina coenda Er. Marcus, 1958.

Distribución. EUA: Florida; México; Belice, Costa Rica, Colombia, Curaçao, Bonaire, Venezuela, Bermudas, Bahamas, islas Caimán, Jamaica, Turku y Caicos, Granada, San Martín, Martinica, Trinidad; Brasil (Valdés et al., 2006; Rosenberg, 2009).

\section{Discusión}

El 61\% de las especies registradas son carnívoras y un $39 \%$ son herbívoras. La mayoría de los ejemplares fueron recolectados sobre sustratos vegetales en la laguna arrecifal de Isla Verde, La Gallega y La Galleguilla durante la época seca del año (de marzo a agosto, Horta-Puga, 1997) (Cuadro 2).

Los estudios sobre moluscos en el golfo de México se han enfocado al registro y análisis comunitario de 
gasterópodos prosobranquios y bivalvos de tallas considerablemente grandes y de fácil captura. Estos trabajos han contribuido ocasionalmente al conocimiento de los opistobranquios, a pesar de que el inventario del grupo se considera incompleto.

Para la región noroeste del océano Atlántico, desde Florida hasta Trinidad y Tobago, se conocen aproximadamente 370 especies de opistobranquios (Valdés et al., 2006); de las cuales al menos 60 representan registros potenciales para el PNSAV (según listas de especies publicadas por Valdés et al., 2006; Bertsch, 2009; Camacho-García, 2009). En contraste, sólo se conocen las 23 especies de opistobranquios que aquí se registran, lo cual da idea de la necesidad de inventarios faunísticos y monitoreos de especies de éstos y otros invertebrados en el PNSAV.

Las especies de opistobranquios del Atlántico oeste son frecuentemente registradas en el intervalo de distribución "Florida a Texas" y "mar Caribe a Brasil". La exclusión del golfo de México origina que éste sea considerado como un vacío taxonómico y biogeográfico debido a la falta de conocimiento sobre la diversidad de sus ecosistemas y especies. Para dar una idea más amplia de esto, se pueden analizar los datos de especies conocidas para la región de Florida a Texas, mar Caribe y Brasil en relación con la fauna total de opistobranquios del Atlántico oeste y del mundo (Cuadro 3). Estos datos ubican al Atlántico oeste como la región del planeta más biodiversa en cefalaspídeos, sacoglosos y anaspídeos, aun sin considerar las especies nuevas o nuevos registros del golfo de México.

En este trabajo se registran por primera vez para el PNSAV 9 especies. El cefalaspídeo Haminoea elegans es un opistobranquio con concha más o menos bien calcificada que habita en las raíces del pasto marino Thalassia testudinum o en zonas con arena más o menos bien protegida. Este animal es común en los meses de abril a agosto. Oxynoe antillarum (Fig. 2J) se mimetiza exitosamente entre los racimos del alga Caulerpa spp, de la cual también se alimenta. Elysia subornata vive entre los tallos de T. testudinum o en la base de las algas verdes Penicillus spp. y Ulva fasciata, de las cuales succiona los fluidos vegetales con ayuda de su único diente radular. Dolabrifera dolabrifera es un opistobranquio herbívoro que puede observarse debajo de rocas en la laguna o cerca de la cresta arrecifal. Stylocheilus longicaudus y $S$. striatus son especies con distribución cosmopolita, se alimentan de algas verdes o pardas y en algunas ocasiones se las puede encontrar en muestras de sargazo flotante. Discodoris evelinae (Fig. 2G), Spurilla neapolitana (Fig. 2F) y Dondice occidentalis son nudibranquios que pueden encontrarse debajo de rocas o sobre corales donde se alimentan de esponjas, anémonas y briozoos.
Los cefalaspídeos Japonacteon punctostriatus, Acteocina canaliculata, A. candei, Volvulella persimilis, Ringicula semistriata, $H$. antillarum y $H$. succinea representan registros casuales en listas de gasterópodos del PNSAV. Estas especies poseen conchas pequeñas (algunas menores a $1 \mathrm{~mm}$ ), muy frágiles y en ocasiones translúcidas, lo cual dificulta su registro si no se realizan muestreos dedicados a este tipo de organismos. Existen muy pocos trabajos enfocados a los micromoluscos del PNSAV y ninguno de ellos ha hecho énfasis en las especies de cefalaspídeos; esta tarea debe promoverse en el futuro.

Navanax aenigmaticus es otro cefalaspídeo poco mencionado en las listas de moluscos del PNSAV; posee una concha frágil, descalcificada e interna; por lo que es imposible su registro si no se le recolecta vivo y durante la época de mayor abundancia y talla (marzo a agosto). Esta especie fue recolectada en fondos arenosos, entre los tallos de T. testudinum y sobre el alga parda Gracillaria blodgetti. El registro de $N$. aenigmaticus sobre $G$. blodgetti es relevante debido a que anteriormente sólo se conocía su presencia sobre arena, debajo de pequeños fragmentos de coral y entre pastos marinos, sustratos en los que se le puede ver buscando activamente pequeñas babosas de los géneros Haminoea y Bulla.

Los aplisiomorfos Aplysia brasiliana, A. dactylomela, A. juliana, Bursatella leachii pleii y el sacogloso E. crispata son los opistobranquios más comunes en el PNSAV. Se les puede ver durante todo el año, aunque de marzo a agosto presentan las tallas y abundancias mayores.

Durante abril y mayo pueden observarse individuos de $A$. dactylomela copulando en largas cadenas de varios individuos o desplazándose a distancias considerablemente grandes (obs. pers.).

Cuadro 3. Comparación de la fauna de opistobranquios en distintas regiones biogeográficas del Atlántico oeste y del mundo (datos tomados de Bertsch, 2009)

\begin{tabular}{|c|c|c|c|c|c|}
\hline 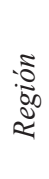 & 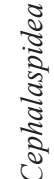 & 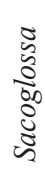 & 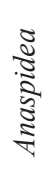 & 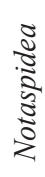 & 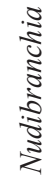 \\
\hline
\end{tabular}

\begin{tabular}{lccccc}
\hline Atlántico oeste & & & & & \\
Oeste Boreal & $46.9 \%$ & $7.3 \%$ & $4.2 \%$ & $3.1 \%$ & $38.5 \%$ \\
Caribe & $29 \%$ & $15.6 \%$ & $5.2 \%$ & $4.6 \%$ & $45.7 \%$ \\
Brasil & $25.9 \%$ & $10 \%$ & $6.3 \%$ & $5.4 \%$ & $50.7 \%$ \\
Indo-Pacífico & $10.7 \%$ & $8.7 \%$ & $1.4 \%$ & $1.2 \%$ & $77 \%$ \\
Atlántico Este & $25.9 \%$ & $6.8 \%$ & $2.9 \%$ & $2.9 \%$ & $61.6 \%$ \\
Atlántico Oeste & $33.9 \%$ & $11 \%$ & $4.4 \%$ & $4.4 \%$ & $45 \%$ \\
Pacífico Oriental & $18 \%$ & $7.6 \%$ & $4.0 \%$ & $4.0 \%$ & $66 \%$ \\
\hline
\end{tabular}


Bulla occidentalis (Fig. 2E) con frecuencia se nombra como B. striata en las listas de gasterópodos del PNSAV (Cruz-Ábrego et al., 1994; De la Cruz y González-Gándara, 2007; Flores-Andolais et al, 1998; García-Cubas, y Reguero, 1990, García-Cubas, Reguero y Elizarraras, 1992; PérezRodríguez, 1997; Quintana, 1991; Reguero, 1989; Reguero y García-Cubas, 1989 y 1993 y Zamora y Naranjo, 2008); después de la revisión sistemática hecha por Malaquias y Reid (2008), la prevalencia del uso de B. occidentalis sólo se considera para ejemplares del Atlántico oeste.

El registro de 23 especies de opistobranquios en el PNSAV es un avance significativo para el conocimiento de la malacofauna de ecosistemas del golfo de México. Sin embargo, se debe reconocer que el número de especies ahora conocido sigue siendo escueto y que se requieren nuevos esfuerzos para conocer éstas y otras especies del PNSAV antes de que el deterioro ambiental las extinga localmente.

\section{Agradecimientos}

A las directoras de las tesis de licenciatura que derivaron en esta publicación: Dra. Martha Reguero-Reza y M. en C. Rosa Estela Toral-Almazán. A Ángel Valdés y Manuel Malaquias por permitirnos usar algunas de sus fotografías incluidas en este artículo. A Tania Islas, Alya Ramos Ramos-Elorduy, Cecilia González, Rebeca Salcedo, Daniel Gómez, Sandra Martínez-Rentería y a los alumnos y profesores del taller "Inventario y monitoreo de la biodiversidad de los arrecifes coralinos del Parque Nacional Sistema Arrecifal Veracruzano" por su apoyo en los muestreos en campo.

\section{Literatura citada}

Andrews, J. 1971. Sea shells of the Texas coast. University of Texas Press, Texas. 298 p.

Angulo-Campillo, O. 2002. New distributional records of opisthobranch molluses from the Gulf of California, Mexico. The Festivus 34:117-121.

Ávila, C. 1992. A preliminary catalogue of natural substances of opisthobranch mollusks from western Mediterranean and near Atlantic. Scientia Marina 56:373-382.

Bertsch, H. 2009. Book review of Indo-Pacific nudibranchs and sea slugs (Gosliner, Behrens y Valdés, 2008), with comparisons of global and Indo-Pacific opisthobranch taxonomic biodiversity and biogeography. Opisthobranch Newsletter 30:1-9.

Bouchet, P. y J. Rocroi. 2005. Classification and nomenclator of gastropod families. Malacologia 47:1-357.

Camacho-García, Y. 2009. Benthic opisthobranchs. In Marine Biodiversity of Costa Rica, Central America, I. S. Wehrtmann y J. Cortés (eds.). Springer Science, Nueva
York. p. 371-386.

Cruz-Ábrego, F. M., A. Toledano-Granados y F. FloresAndolais. 1994. Ecología comunitaria de los gasterópodos marinos (Mollusca: Gastropoda) en isla Contoy. Revista de Biología Tropical 42:549-556.

De la Cruz-Francisco, V. y C. González-Gándara. 2007. Lista actualizada de los gasterópodos de la planicie del arrecife Lobos, Veracruz, México. Revista UDO Agrícola 6:128-137.

Ekdale, A. A. 1974. Marine molluscs from the shallow-water environments (0-60 meters) off the northeast Yucatan coast, Mexico. Bulletin of Marine Science 24:638-668.

Flores-Andolais, F., A. García-Cubas y A. Toledano-Granados. 1988. Sistemática y algunos aspectos ecológicos de los moluscos de la laguna de La Mancha, Veracruz, México. Anales del Instituto de Ciencias del Mar y Limnología, Universidad Nacional Autónoma de México 175:235-258.

García-Cubas, A. y M. Reguero. 1990. Moluscos del sistema lagunar Tupilco-Ostión, Tabasco, México: Sistemática y ecología. Anales del Instituto de Ciencias del Mar y Limnología, Universidad Nacional Autónoma de México 17:345-359.

García-Cubas, A., F. Escobar, L. González y M. Reguero. 1990. Moluscos de la Laguna de Mecoacán, Tabasco, México: Sistemática y ecología. Anales del Instituto de Ciencias del Mar y Limnología, Universidad Nacional Autónoma de México 17:309-343.

García-Cubas, A., M. Reguero y R. Elizarrarás. 1992. Moluscos del sistema lagunar Chica-Grande, Veracruz, México: Sistemática y ecología. Anales del Instituto de Ciencias del Mar y Limnología, Universidad Nacional Autónoma de México 19:71-101.

Gosliner, T., Behrens, D. y Á. Valdés. 2008. Indo-Pacific nudibranchs and sea slugs. A field guide to the world's most diverse fauna. Sea Challengers Natural History Books and California Academy of Sciences, San Francisco. 426 p.

Hermosillo, A., D. Behrens y E. Ríos. 2006. Opistobranquios de México. Guía de babosas marinas del Pacíifico, golfo de California y las islas oceánicas. Comisión Nacional para el Conocimiento y Uso de la Biodiversidad, México, D. F. $143 \mathrm{p}$.

Horta-Puga, G. 1997. The Veracruz reef system in the Gulf of México, and environmental review. Resúmenes del Simposio Ecosistemas Acuáticos de México, Faculta de Estudios Superiores Iztacala, Estado de México, México. 1997. $210 \mathrm{p}$.

Malaquias, M, y D. Reid. 2008. Systematic revision of the living species of Bullidae (Mollusca: Gastropoda: Cephalaspidea), with a molecular phylogenetic analisis. Zoological Journal of the Linnean Society 153:453-543.

Moore, D. R. 1958. Notes on Blanquilla Reef, the most northerly coral formation in Western Gulf of Mexico. Institute of Marine Science 5:151-155. 
Pérez-Rodríguez, R. 1997. Moluscos de la plataforma continental del Atlántico mexicano. Universidad Autónoma Metropolitana, México, D. F. 260 p.

Quintana, J. 1991. Resultados del Programa de investigaciones en arrecifes veracruzanos del laboratorio de sistemas bentónicos litorales. Hidrobiología 1:73-79.

Redfern, C. 2001. Bahamian seashells: A thousand species from Abaco, Bahamas. Bahamianseashells.com, Boca Raton, Florida. $280 \mathrm{p}$.

Reguero, M. y A. García-Cubas. 1989. Moluscos de la laguna de Alvarado, Veracruz: sistemática y ecología. Anales del Instituto de Ciencias del Mar y Limnología, Universidad Nacional Autónoma de México 16:279-306.

Reguero, M. y A. García-Cubas. 1993. Moluscos de la laguna Pueblo Viejo, Veracruz, México: Sistemática y ecología. Anales del Instituto de Ciencias del Mar y Limnología, Universidad Nacional Autónoma de México 20:77-104.

Rice, W. H. y L. S. Kornicker. 1962. Mollusks of Alacran Reef, Campeche Bank, Mexico. Publications of the Institute of Marine Science 8:366-403.

Rice, W. H. y L. S. Kornicker. 1965. Mollusks from the deeper waters of the northwestern Campeche Bank, Mexico. Publications of the Institute of Marine Science 10:108-172.

Rosenberg, G. 2009. Malacolog ver. 4.1.0: A database of western Atlantic marine Mollusca. http://www.malacolog. org/; última consulta: 30.X.2011.

Secretaría de Desarrollo Social. 1992. Decreto por el que se declara área natural protegida con el carácter de Parque Marino Nacional, la zona conocida como Sistema Arrecifal
Veracruzano, ubicada frente a las Costas de los municipios de Veracruz, Boca del Río y Alvarado del estado de Veracruz Llave, con superficie de 52,238-91-50 hectáreas. Diario Oficial de la Federación, 24 de agosto, tomo CDLXVII, 16:6-15.

Strenth, N. E. y J. E. Blankenship. 1977. Notes on sea hare of South Texas. Veliger 20:98-100.

Thompson, T. 1976. Biology of opisthobranch molluses. University of Bristol, London. 207 p.

Valdés, Á., J. Hamann, D. Behrens y A. DuPont. 2006. Caribbean Sea slugs. A field guide to the opisthobranchs mollusks from the tropical northwestern Atlantic. Sea Challengers Natural History, Gig Harbour, Washington. 289 p.

Vargas-Hernández, J. M., A. Hernández-Gutiérrez y L. F. Carrera-Parra. 1993. Sistema Arrecifal Veracruzano. In Biodiversidad marina y costera de México, S. SalazarVallejo y N. E. González (comps.).Comisión Nacional para el Conocimiento y Uso de la Biodiversidad/Centro de Investigaciones de Roo, Chetumal. p. 559-575.

Vokes, H. E. y E. H. Vokes. 1983. Distribution of shallowwater marine Mollusca, Yucatan Peninsula, Mexico. Mesoamerican Ecology Institute, Monograph 1. Tulane University, New Orleans. 183 p.

Wiley, G., R. Circé y J. Tunell. 1982. Mollusca of the rocky shores of East Central Veracruz State, Mexico. The Nautilus 96:55-61.

Zamora-Silva, A. y E. Naranjo-García. 2008. Los opistobranquios de la Colección Nacional de Moluscos. Revista Mexicana de Biodiversidad 79:333-342. 